\title{
Editorial
}

Oncology

\section{External Beam Radiation with 'Curative Intent' in Advanced Ovarian Cancer: An Uncommon but Rational Management Approach Based on the Natural History of Cancer in an Individual Patient}

\author{
Maurie Markman \\ Cancer Treatment Centers of America, Eastern Regional Medical Center, Philadelphia, Pa., USA
}

On the surface, it is difficult to think of a setting where the concept of the delivery of external beam radiation with 'curative intent' would be more inappropriate. Advanced ovarian cancer is recognized to be widespread throughout the peritoneal cavity in the large majority of patients at diagnosis.

In fact, the rationale for considering 'stage 2' ovarian cancer as an objectively valid clinical entity has been questioned, suggesting that, in the large majority of cases, one is really dealing with 'stage 3 ' disease even though it is not possible to confirm (during surgery) the presence of micro-metastatic disease in the upper abdomen. Furthermore, the statistically more favorable outcome for such patients (stage 2), compared to women who present with documented metastatic disease outside the pelvis, is likely the result of the presence of a substantially reduced tumor bulk rather than being due to the specific anatomic localization of the cancer.

Yet, Machida et al. [1] in this issue of Oncology and other investigators have reported that a small subset of patients with recurrent ovarian cancer treated with external beam radiation following the failure of cytotoxic chemotherapy may experience very long-term survival (and possibly a 'cure' of the malignant process) [1-3].

Of interest, the fundamental justification for this strategy in a highly selected patient population is actually

\section{KARGER}

E-Mail karger@karger.com

www.karger.com/ocl based on the observations noted above. It is most uncommon for recurrent ovarian cancer to be anatomically localized to the pelvic region, with radiographic imaging studies or surgical exploration providing confirmation for macro- or micro-metastatic cancer within the upper abdomen (or even more extensive spread).

Therefore, when the cancer of an individual patient with ovarian cancer is documented to be present within the pelvis and there is no evidence of disease outside the confines of this anatomic location, it is reasonable to suggest that this particular cancer in this specific patient behaves, biologically, in a manner quite different from what is expected. Moreover, under such circumstances, it is rational to argue that by achieving local disease control in the pelvis (following external beam radiation delivered with the intent of attaining this goal) it is possible the patient may experience a prolonged period of disease-free survival (measured in 'years').

The concept of carefully observing the natural history of a cancer in an individual patient and the increasingly common use of performing molecular profiling of a patient's malignancy to help define relevant driver mutations are both critically relevant components of a therapeutic focus on delivering 'personalized cancer care' that is designed to maximize the opportunity of a favorable clinical outcome.
C 2014 S. Karger AG, Basel

0030-2414/14/0864-0230\$39.50/0
Maurie Markman, MD

Cancer Treatment Centers of America, Eastern Regional Medical Center 1331 East Wyoming Avenue Philadelphia, PA 19124 (USA)

E-Mail maurie.markman@ctca-hope.com 
References
1 Machida S, Takei Y, Yoshida C, Takahashi Y, Koyanagi T, Sato N, Taneichi A, Saga Y, Fujiwara $\mathrm{H}$, Suzuki M: Radiation therapy for chemotherapy-resistant recurrent epithelial ovarian cancer. Oncology 2014;86:232-238.

2 Brown AP, Jhingran A, Klopp AH, et al: Involved-field radiation therapy for locoregionally recurrent ovarian cancer. Gynecol Oncol 2013;130:300-305.
3 Davidson SA, Rubin SC, Mychalczak B, et al: Limited-field radiotherapy as salvage treatment of localized persistent or recurrent epithelial ovarian cancer. Gynecol Oncol 1993; 51:349-354. 